\section{Tocilizumab ist zur Be- handlung des Primären Sjögren Syndroms ungeeignet}

Felten $\mathrm{R}$ et al. Interleukin 6 receptor inhibition in primary Sjögren syndrome: a multicentre double-blind randomized placebo-controlled trial. Ann Rheum Dis 2021; 80: 329-338

Bis heute gibt es kein Medikament, für das eine Wirksamkeit zur Behandlung des primären Sjögren Syndroms (pSS) gezeigt wurde. Es wird vermutet, dass Interleukin 6 (IL-6) mit seiner entscheidenden Rolle bei der B-Zell-Aktivierung und T-Zell-Polarisation eine wichtige pathogene Rolle bei pSS spielt. Felten et al. untersuchten die Wirkung von Tocilizumab bei Patienten mit pSS mit moderater oder hoher Krankheitsaktivität.

Die Anwendung von Tocilizumab führte bei Patienten mit pSS im Vergleich zu Placebo über einen Zeitraum von 24 Wochen zu keiner Verbesserung der systemischen Manifestation. Die Wissenschaftler rekrutierten aus insgesamt 17 klinischen Zentren in Frankreich zwischen Juli 2013 und Juni 2017 Patienten mit pSS gemäß den Kriterien der American European Consensus Group und ein Score $\geq 5$ für den EULAR Sjögren's Syndrome Disease Activity Index (ESSDAI). Die Experten randomisierten die Patienten in einem Verhältnis von 1:1 und verabreichten entweder 6 monatliche Infusionen Tocilizumab $(8 \mathrm{mg} / \mathrm{kg})$ oder ein Placebo zwischen Woche 0 und Woche 20. Die Experten wendeten ein dreifach-verblindetes Verfahren an, wobei neben dem Studienleiter und den Patienten auch der Statistiker während der Analysen keine Kenntnis über die zugewiesene Behandlungsgruppe hatte. Den primären Endpunkt ermittelten die Forscher nach Woche 24. Nach Absetzen des Medikamentes untersuchten die Ärzte die Patienten nach Woche 32 und 44. Ein Ansprechen auf die Behandlung definierten die Experten als die Abnahme des ESSDAI um mindestens 3 Punkte, kein Auftreten von moderater oder schwerer Aktivität in einer neuen Domäne des ESSDAI und keine Verschlechterung der ärztlichen Gesamtbeurteilung auf einer visuellen numerischen Skala von 1-10, jeweils im Vergleich zum Studienbeginn.
Die Experten randomisierten 110 Patienten: 55 Patienten teilten sie der Tocilizumab-Gruppe und 55 der Placebo-Gruppe zu. Nach 24 Wochen betrug der Anteil der Patienten, die den primären Endpunkt erreichten, lediglich $52,7 \%$ in der Tocilizumab-Gruppe und 63,6\% in der PlaceboGruppe. Der durchschnittliche ESSDAI in Woche 24 betrug 8,3 in der Tocilizumabund 7,2 in der Placebo-Gruppe. Post-hocAnalysen zeigten, dass Tocilizumab bei Patienten mit moderater oder hoher systemischer Krankheitsaktivität im Vergleich zu Placebo keine bessere Wirksamkeit aufwies. Zwischen Woche 0 und Woche 44 traten insgesamt 15 SAEs in der TocilizumabGruppe und 11 in der Placebo-Gruppe auf.

\section{FAZIT}

Die Resultate der vorliegenden Studie zeigen, dass die Anwendung von Tocilizumab im Vergleich zu Placebo bei Patienten mit einem Primären Sjögren Syndrom nicht zu einer Verbesserung der systemischen Beteiligung oder der Symptome über 24 Behandlungswochen führt. Diese Ergebnisse deuten darauf hin, dass IL-6 kein relevantes therapeutisches Ziel beim pSS darstellt, so die Experten.

Dr. Maddalena Angela Di Lellis, Tübingen 